\title{
ALMA OBSERVATIONS OF THE LARGEST PROTO-PLANETARY DISK IN THE ORION NEBULA, 114-426: A CO SILHOUETTE
}

\author{
John Bally $^{1}$, Rita K. Mann ${ }^{2}$, Josh Eisner ${ }^{3}$, Sean M. Andrews ${ }^{4}$, James Di Francesco ${ }^{2,5}$, Meredith Hughes ${ }^{6}$, \\ Doug Johnstone $^{2,5}$, Brenda Matthews ${ }^{2,5}$, Luca Ricci ${ }^{7}$, and Jonathan P. Williams ${ }^{8}$ \\ ${ }^{1}$ Department of Astrophysical and Planetary Sciences, University of Colorado, UCB 389, Boulder, CO 80309, USA; john.bally@colorado.edu \\ 2 National Research Council Canada, 5071 West Saanich Road, Victoria, BC, V9E 2E7, Canada \\ ${ }^{3}$ Steward Observatory, University of Arizona, 933 North Cherry Avenue, Tucson, AZ 85721, USA \\ ${ }_{5}^{4}$ Harvard-Smithsonian Center for Astrophysics, 60 Garden Street, Cambridge, MA 02138, USA \\ 5 Department of Physics and Astronomy, Uiversity of Victoria, Victoria, BC, V8P 1A1, Canada \\ ${ }^{6}$ Van Vleck Observatory, Astronomy Department, Wesleyan University, 96 Foss Hill Drive, Middletown, CT 06459, USA \\ ${ }^{7}$ Department of Astronomy, California Institute of Technology, MC 249-17, Pasadena, CA 91125, USA \\ Institute for Astronomy, University of Hawaii, Honolulu, HI 96816, USA \\ Received 2015 April 30; accepted 2015 June 8; published 2015 July 17
}

\begin{abstract}
We present ALMA observations of the largest protoplanetary disk in the Orion Nebula, 114-426. Detectable $345 \mathrm{GHz}(856 \mu \mathrm{m})$ dust continuum is produced only in the $350 \mathrm{AU}$ central region of the $\sim 1000$ AU diameter silhouette seen against the bright $\mathrm{H} \alpha$ background in Hubble Space Telescope images. Assuming optically thin dust emission at $345 \mathrm{GHz}$, a gas-to-dust ratio of 100 , and a grain temperature of $20 \mathrm{~K}$, the disk gas-mass is estimated to be $3.1 \pm 0.6$ Jupiter masses. If most solids and ices have been incorporated into large grains, however, this value is a lower limit. The disk is not detected in dense-gas tracers such as $\mathrm{HCO}^{+} J=4-3$, $\mathrm{HCN} J=4-3$, or CS $=7-6$. These results may indicate that the 114-426 disk is evolved and depleted in some light organic compounds found in molecular clouds. The $\mathrm{CO} J=3-2$ line is seen in absorption against the bright $50-80 \mathrm{~K}$ background of the Orion A molecular cloud over the full spatial extent and a little beyond the dust continuum emission. The CO absorption reaches a depth of $27 \mathrm{~K}$ below the background $\mathrm{CO}$ emission at $V_{\mathrm{LSR}} \approx 6.7 \mathrm{~km} \mathrm{~s}^{-1} \sim 0^{\prime \prime} .52(210$ AU) northeast and $12 \mathrm{~K}$ below the background $\mathrm{CO}$ emission at $V_{\mathrm{LSR}} \approx 9.7 \mathrm{~km} \mathrm{~s}^{-1} \sim 0^{\prime \prime} .34(140 \mathrm{AU})$ southwest of the suspected location of the central star, implying that the embedded star has a mass less than $1 M_{\odot}$.
\end{abstract}

Key words: circumstellar matter - protoplanetary disks - stars: pre-main sequence

\section{INTRODUCTION}

The Orion Nebula contains over a thousand low-mass young stars, many of which are surrounded by protoplanetary disks (O'Dell 2001). UV radiation from the massive Trapezium stars is photo-ablating these disks to produce several 100 proplyds with comet-shaped, ionized skins seen in $\mathrm{H} \alpha$ and visual-wavelength forbidden emission-line images (O’Dell \& Wen 1994; Bally et al. 2000). In addition to these bright proplyds, the Nebula contains several dozen disks seen in silhouette against the bright background of nebular light. The absence of detectable emission lines from some silhouettes suggests that they are either located far from the Orion Nebula's ionizing stars or completely outside the $\mathrm{H}$ II region in the foreground.

With a major axis diameter of $2 " .3$, or $950 \mathrm{AU}$ at a distance of $414 \mathrm{pc}$ (Menten et al. 2007), the 114-426 disk is the largest silhouette in the Orion Nebula (McCaughrean \& O'Dell 1996). Throop et al. (2001) found that the translucent, northeastern rim has gray extinction to a wavelength of about $2 \mu \mathrm{m}$ implying that grains with sizes of about $1 \mu \mathrm{m}$ or a little less dominate the extinction. Reddening between 1.87 and $4.05 \mu \mathrm{m}$, however, suggests that most of the obscuration is produced by grains smaller than $4 \mu \mathrm{m}$ (Shuping et al. 2003). Miotello et al. (2012) argued that despite being a silhouette, UV-induced photoevaporation of the disk (Johnstone et al. 1998) may be responsible for its apparent warp, the translucent northeastern rim, and the large-bow shaped $\mathrm{H} \alpha$ arc $6^{\prime \prime}-10^{\prime \prime}$ west of the disk (see Figure 1).

The nearly edge-on disk completely obscures its central star whose presence is indicated by a bipolar reflection nebula at visual and near-IR wavelengths. McCaughrean et al. (1998) used extinction of background nebular light to estimate a lower-bound of $2 \times 10^{-4} M_{\odot}$ for the mass of the 114-426 disk. They also used the flux of scattered light in the reflection nebula to infer the intrinsic $K$-band magnitude of the central star, $m_{K} \sim 9.5$, and argued that it is likely to have a mass of $\sim 1.5 M_{\odot}$. The extinction to the star was used to argue for a disk mass larger than $5 \times 10^{-4} M_{\odot}$, higher than the lower bound based on extinction of nebular light. Multiple searches for dust continuum emission at millimeter and submillimeter wavelengths, however, have failed to detect emission (Bally et al. 1998; Williams et al. 2005; Eisner \& Carpenter 2006; Eisner et al. 2008). Mann \& Williams $(2009,2010)$ measured the dust masses of several dozen proplyds with the SMA interferometer, but failed to detect 114-426, placing a limit of $<1.2 \times 10^{-2} M_{\odot}$ on the disk mass. Finally, Mann et al. (2014) detected the dust continuum at $856 \mu \mathrm{m}$ with ALMA, finding a disk mass of about 3.4 Jupiter masses $\left(3.2 \times 10^{-3} M_{\odot}\right)$.

In this paper, we present the ALMA observations of the 114-426 disk in four spectral lines and re-analyze the dust continuum in more detail than Mann et al. (2014). Having a diameter of $\sim 950 \mathrm{AU}, 114-426$ is the largest candidate protoplanetary disk seen in silhouette against the Orion Nebula. Nevertheless, the $856 \mu \mathrm{m}$ continuum is confined to the inner $\sim 350 \mathrm{AU}$ region. Remarkably, carbon monoxide, the only molecule detected in the 114-426 disk, is observed in absorption against the warm background $\mathrm{CO}$ emission from the Orion A molecular cloud. The central star's mass is constrained by the disk rotation curve to be less than $1 M_{\odot}$. The observations show high-velocity $\mathrm{CO}$ 


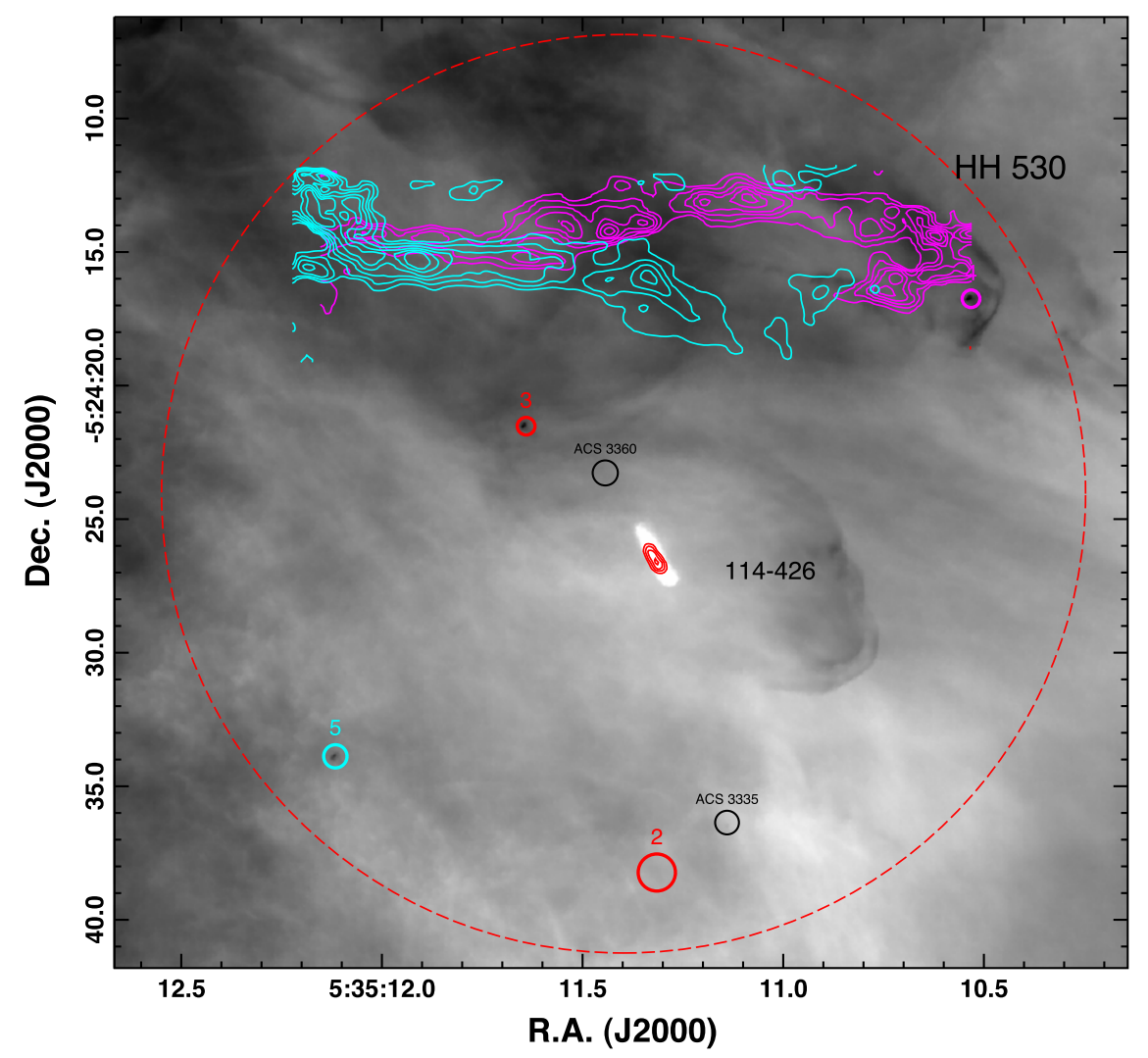

Figure 1. HST H $\alpha$ image of the 114-426 field (Object 1 in Table 1). The large dashed circle shows the ALMA primary beam field of view at 350 GHz. The numbered sources mark objects listed in Table 1 (except 114-426). The red circles show two objects $(2,3)$ with submillimeter continuum detections in addition to the 114-426 disk. The cyan circle marks the location of object $5(121-434=$ OW94 = V2224 Ori), which was not detected by ALMA. The two black circles mark additional stars detected in broadband HST images taken as part of the HST Treasury Program on the Orion Nebula Cluster in Cycle 13, GO Program 10246, P. I.: M. Robberto (Ricci et al. 2008; Robberto et al. 2013). The CO outflow associated with HH 530 discussed in the text is shown in magenta and cyan contours. Cyan contours show blueshifted emission in the radial velocity range $V_{\mathrm{LSR}}=2-5.9 \mathrm{~km} \mathrm{~s}^{-1}$. Magenta contours show redshifted emission in the radial velocity range $V_{\mathrm{LSR}}=10.5-14.3 \mathrm{~km} \mathrm{~s}{ }^{-1}$. Contour levels are at $0.1,0.2,0.3,0.4,0.5$, and $0.6 \mathrm{Jy} \mathrm{beam}^{-1}$. A magenta circle an arcsecond below the western end of the CO flow marks the location of object 4 in the table, V2202 Ori. The red contours superimposed on the image of the 114-426 disk show the $856 \mu$ m dust continuum emission with levels at $1,2,3$, and 4 mJy beam ${ }^{-1}$.

emission $\sim 10^{\prime \prime}$ north of $114-426$, possibly associated with the $\mathrm{HH}$ 530 protostellar outflows in the Orion Nebula.

\section{OBSERVATIONS}

The data analyzed here were obtained with ALMA during Cycle 0 (ALMA project 2011.0.00028.S) on 2012 October 24 using twenty-two $12 \mathrm{~m}$ antennas, as part of a study of the Orion proplyds. Mann et al. (2014) present a detailed description of the observations and data reduction procedures. That paper also discusses the disk dust masses measured using the $856 \mu \mathrm{m}$ $(350.0 \mathrm{GHz})$ dust continuum. In this paper, we present the molecular line data for the silhouette disk, 114-426. Four transitions were observed in ALMA Band 7; CO $J=3-2$, $\mathrm{HCO}^{+} J=4-3, \mathrm{HCN} J=4-3$, and CS $J=7-6$. Total on-source integration times were $1300 \mathrm{~s}$, resulting in a continuum sensitivity of about $0.56 \mathrm{mJy}$ per beam. The synthesized beam FWHM size is $0 \prime$.51 ( 211 AU) $\times 00^{\prime \prime} .46(\sim 190 \mathrm{AU})$. At this frequency, $1 \mathrm{Jy}$ corresponds to a brightness temperature of $42.8 \mathrm{~K}$ in the synthesized beam. The primary-beam field of view (FOV) is $18^{\prime \prime}$ and the maximum recoverable angular structure is about $5^{\prime \prime}(\sim 2000 \mathrm{AU})$. The correlator was configured to observe four $1.875 \mathrm{GHz}$ wide bands using a channel spacing of $488.28 \mathrm{kHz}$ to yield 3840 channel spectra. After Hanning smoothing, the spectral resolution was $\Delta \nu=976.56 \mathrm{kHz}$ corresponding to a velocity resolution $\Delta V=0.84 \mathrm{~km} \mathrm{~s}^{-1}$. The spectral line sensitivity of these observations is limited, presumably by over-resolved fluctuations in the spatial and radial velocity structure of the spectral-line emission from the background molecular cloud, to about $0.025-0.1 \mathrm{Jy}^{\text {beam }}{ }^{-1}$ per Hanning-smoothed channel, or about $1-4 \mathrm{~K}$ per channel.

The reduced data were re-gridded to $X_{\text {pix }}=0^{\prime \prime}$. 11 per pixel. Each pixel value corresponds to the flux that would be measured by the ALMA synthesized beam at the pixel center. The number of square pixels with dimension $X_{\text {pix }}$ in a circular aperture which has the same effective area on the sky as a Gaussian beam with a (circular) FWHM $=0$ ". 485 is given by $N_{\text {pix }}=[\pi / 4 \ln (2)]\left(\mathrm{FWHM} / X_{\text {pix }}\right)^{2} \approx 22.03$ pixels. ${ }^{9}$ Thus, the total flux in an aperture containing $n$ pixels is obtained by summing the pixel values in the aperture and dividing by $N_{\text {pix }}$.

\section{RESULTS}

Figure 1 shows the FOV of the ALMA $856 \mu \mathrm{m}$ primary beam (large dashed circle) superimposed on a Hubble Space Telescope (HST) H $\alpha$ image (Bally et al. 2006). Red contours show the dust continuum emission from the 114-426 disk on the visual wavelength silhouette. High-velocity $\mathrm{CO}$ emission from a background protostellar outflow suspected to be

\footnotetext{
9 In terms of the FITS keywords BMAJ, BMIN, CDELT1, and CDELT2, the quantity $\left(\mathrm{FWHM} / X_{\mathrm{pix}}\right)^{2}$ is given by $(B M A J * B M I N) /$ $(C D E L T 1 * C D E L T 2)$.
} 

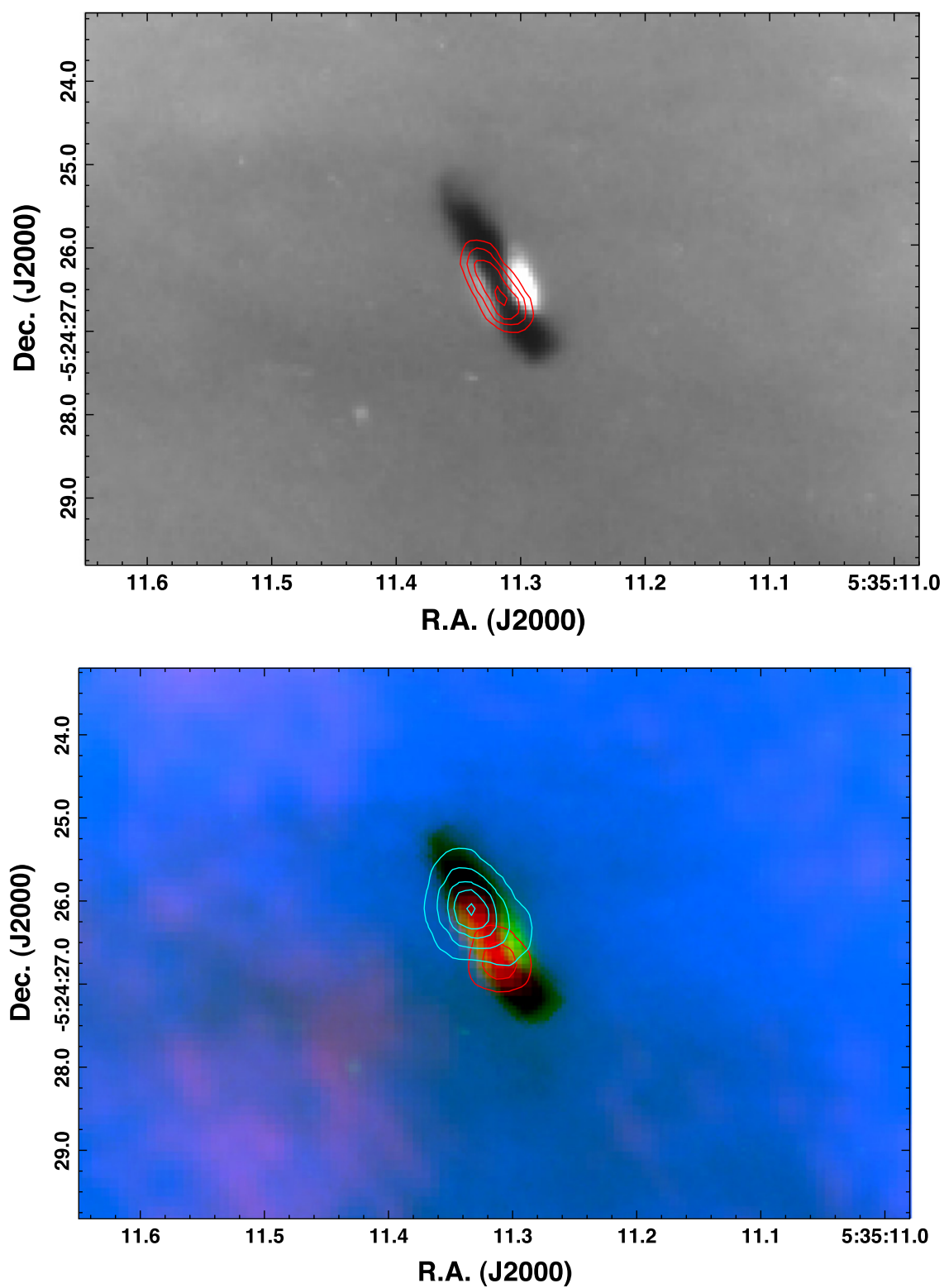

Figure 2. Top: a closeup showing an F775W image from the Hubble Space Telescope (Ricci et al. 2008) with contours of $856 \mu \mathrm{m}$ dust continuum emission superimposed. Contour levels are at 1,2,3, and $4 \mathrm{mJy}_{\text {beam }}{ }^{-1}$. Bottom: a closeup of the 114-426 disk showing the H $\alpha$ image (blue) and the F775W image (green) from the Hubble Space Telescope. The dust continuum emission at $856 \mu \mathrm{m}$ is shown (red) superimposed on the HST image. Contours of redshifted and blueshifted CO $J=3-2$ absorption at $V_{\mathrm{LSR}}=7.13 \mathrm{~km} \mathrm{~s}^{-1}$ (cyan contours) and at $V_{\mathrm{LSR}}=9.24 \mathrm{~km} \mathrm{~s}^{-1}$ (red contours), each in $0.42 \mathrm{~km} \mathrm{~s}^{-1}$ wide channels are shown. Contour levels are at $-0.2,-0.3,-0.4,-0.5$, and $-0.6 \mathrm{Jy} \mathrm{beam}^{-1}$.

associated with Herbig-Haro (HH) object HH 530 is shown in cyan and magenta contours.

Figure 2 (top) shows a closeup of the disk in the F775W HST filter (Ricci et al. 2008; Robberto et al. 2013) with superimposed contours of $856 \mu \mathrm{m}$ continuum emission. The submillimeter dust emission has a major axis FWHM diameter of $1^{\prime \prime} .11 \pm 0$ ". 05 , and a minor axis FWHM diameter of 0 ". $53 \pm$ 0 ". 05 . The beam de-convolved ${ }^{10}$ FWHM disk major and minor axis diameters are $1^{\prime \prime} .0(\sim 414 \mathrm{AU})$ and less than 0 ". $2(<80 \mathrm{AU})$, implying that it is unresolved along its minor axis. Thus, the disk outer radius at $856 \mu \mathrm{m}$ is about 0 ".5 $(200 \mathrm{AU})$, more than a factor of two smaller than the

\footnotetext{
${ }^{10}$ The de-convolved major and minor axes were estimated by taking the square-root of the difference of the squares of the observed FWHM and beam FWHM.
}

visual wavelength radius of $475 \mathrm{AU}$. The peak flux, $4.2 \mathrm{mJy}$ beam $^{-1}$ is located at $\mathrm{J} 2000=05: 35: 11.316,-5: 24: 26.62$, coincident with the expected position of the central star based on the symmetry of the bipolar reflection nebula seen in the HST F775W filter image (Figure 2). The area integrated flux in a $\sim 0$ ". 74 by 1 1". 55 diameter elliptical aperture aligned with the disk major axis and centered on the emission is $6.0 \pm 0.6 \mathrm{mJy}$, $14 \%$ smaller than the $7 \mathrm{mJy}$ flux reported by (Mann et al. 2014). The two flux measurements differ slightly because of variations in the size and shape of the measurement aperture combined with the presence of a low-amplitude negative bowl surrounding the continuum source. The disk mass is computed from the measured flux density, $S_{\nu}$, the distance $D=414 \mathrm{pc}$ (Mann et al. 2014 used a distance of $400 \mathrm{pc}$ ), a grain emissivity $\kappa=0.034 \mathrm{~cm}^{2} \mathrm{~g}^{-1}$ (which assumes a gas-to-dust ratio of 100 ), 
Table 1

Sources in the d114-426 Field

\begin{tabular}{|c|c|c|c|c|}
\hline No. & $\begin{array}{c}\alpha \\
\text { J2000 }\end{array}$ & $\begin{array}{c}\delta \\
\mathrm{J} 2000\end{array}$ & $\begin{array}{l}\text { Flux }^{a} \\
(\mathrm{mJy})\end{array}$ & Comments \\
\hline 1 & 053511.32 & -052426.4 & $6.0 \pm 0.6$ & $114-426$ \\
\hline 2 & 053511.32 & -052438.2 & $88 \pm 10$ & 2MASS 05351131-0524381 ${ }^{\mathrm{b}}$ \\
\hline 3 & 053511.66 & -052421.5 & $3.7 \pm 0.6$ & OW94 117-421; 2MASS 05351165-0524213 \\
\hline 4 & 053510.53 & -052416.8 & $<4$ & OW94 106-417; HH 530; V2202 Ori \\
\hline 5 & 053512.11 & -052433.9 & $<9$ & OW94 121-434; V2224 Ori \\
\hline
\end{tabular}

Note.

${ }^{\mathrm{a}}$ Fluxes are measured on the ALMA dust continuum image.

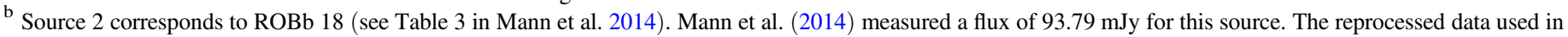
this paper gives a slightly lower flux. The discrepancy is probably due to the fact that the star is at the edge of the primary beam.
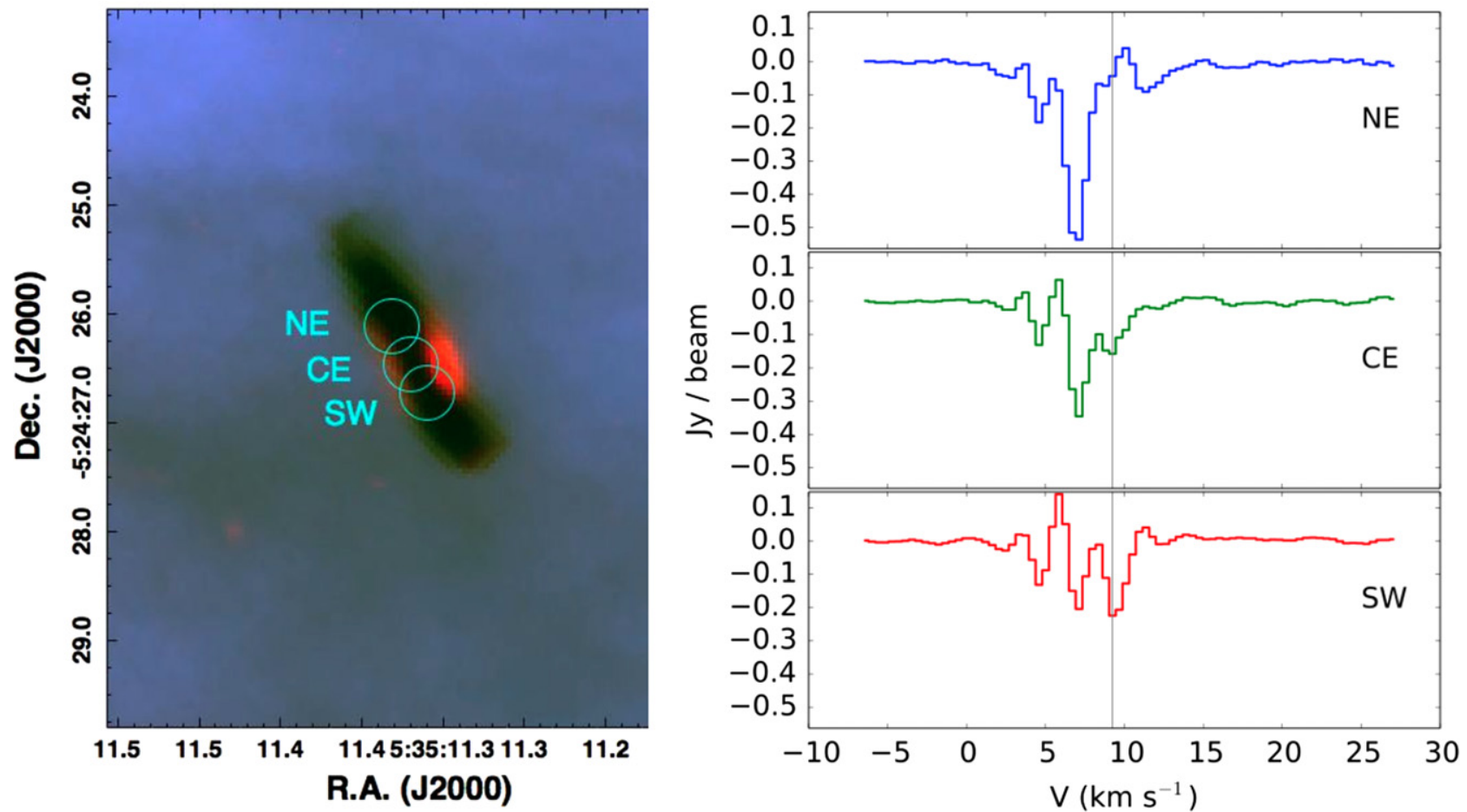

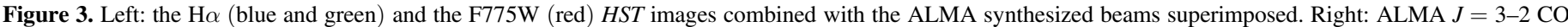

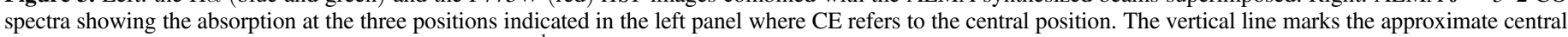
velocity of the background emission, $V_{\mathrm{LSR}}=9.2 \mathrm{~km} \mathrm{~s}^{-1}$.

and a grain temperature $T_{\text {dust }}=20 \mathrm{~K}$, using the formula

$$
M_{\text {disk }}=\frac{S_{\nu} D^{2}}{\kappa B_{\nu}\left(T_{\text {dust }}\right)} \approx 5.9 \pm 1.1 \times 10^{30} \mathrm{~g},
$$

where $B_{\nu}\left(T_{\text {dust }}\right)$ is the Planck function. Using these values, the disk mass is only $3.1 \pm 0.6$ times the mass of Jupiter.

Williams \& Best (2014) presented a parametric model for the estimation of disk gas masses using isotopologues of CO. They found that for nine disks they studied, the mass estimates based on the dust continuum flux using a standard gas-to-dust ratio of 100 were systematically higher than the masses based on $\mathrm{CO}$ isotopologues. Future ALMA observations of $\mathrm{CO}$ isotopologues should be obtained to test the validity of the assumptions used in converting the submillimeter continuum flux into a disk gas mass.

Remarkably, the 114-426 disk is seen in absorption in the ALMA ${ }^{12} \mathrm{CO} J=3-2$ images (Figures 2 (Bottom), 3, and 4 and Table 2). The background $\mathrm{CO}$ emission from the Orion $\mathrm{A}$ cloud is bright. In the Nobeyama $45 \mathrm{~m} 15^{\prime \prime}$ telescope beam centered on $114-426$, the ${ }^{12} \mathrm{COJ}=1-0$ emission has a brightness temperature above $50 \mathrm{~K}$ between $V_{\mathrm{LSR}}=5$ and $10 \mathrm{~km} \mathrm{~s}^{-1}$ with a peak value of $82 \mathrm{~K}$ at $V_{\mathrm{LSR}}=8.0 \mathrm{~km} \mathrm{~s}^{-1}$ (Shimajiri et al. 2011, 2014). ${ }^{11}$ The IRAM $30 \mathrm{~m}^{12} \mathrm{CO} J=2-1$ emission (Berne et al. 2014) shows peak temperatures of 60 $-70 \mathrm{~K}$ and the APEX ${ }^{12} \mathrm{CO} J=3-2$ map (Peng et al. 2012) shows peak temperatures of around $70 \mathrm{~K}$ behind $114-426 .^{12}$ These CO lines are optically thick as indicated by the high intensities of the ${ }^{13} \mathrm{CO}$ and $\mathrm{C}^{18} \mathrm{O}$ lines at this location.

The ALMA interferometer filters-out structure on spatial scales larger than $\sim 5^{\prime \prime}$ in the configuration that was used for these observations. Thus, the mean total power in each

\footnotetext{
11 The Nobeyama data was downloaded from the NRO Star Formation Legacy Project website at http://th.nao.ac.jp/MEMBER/nakamrfm/sflegacy/ sflegacy.html.

12 Since we do not have access to these data in digital form, our analysis relies on extrapolation of the lower-lying transitions.
} 


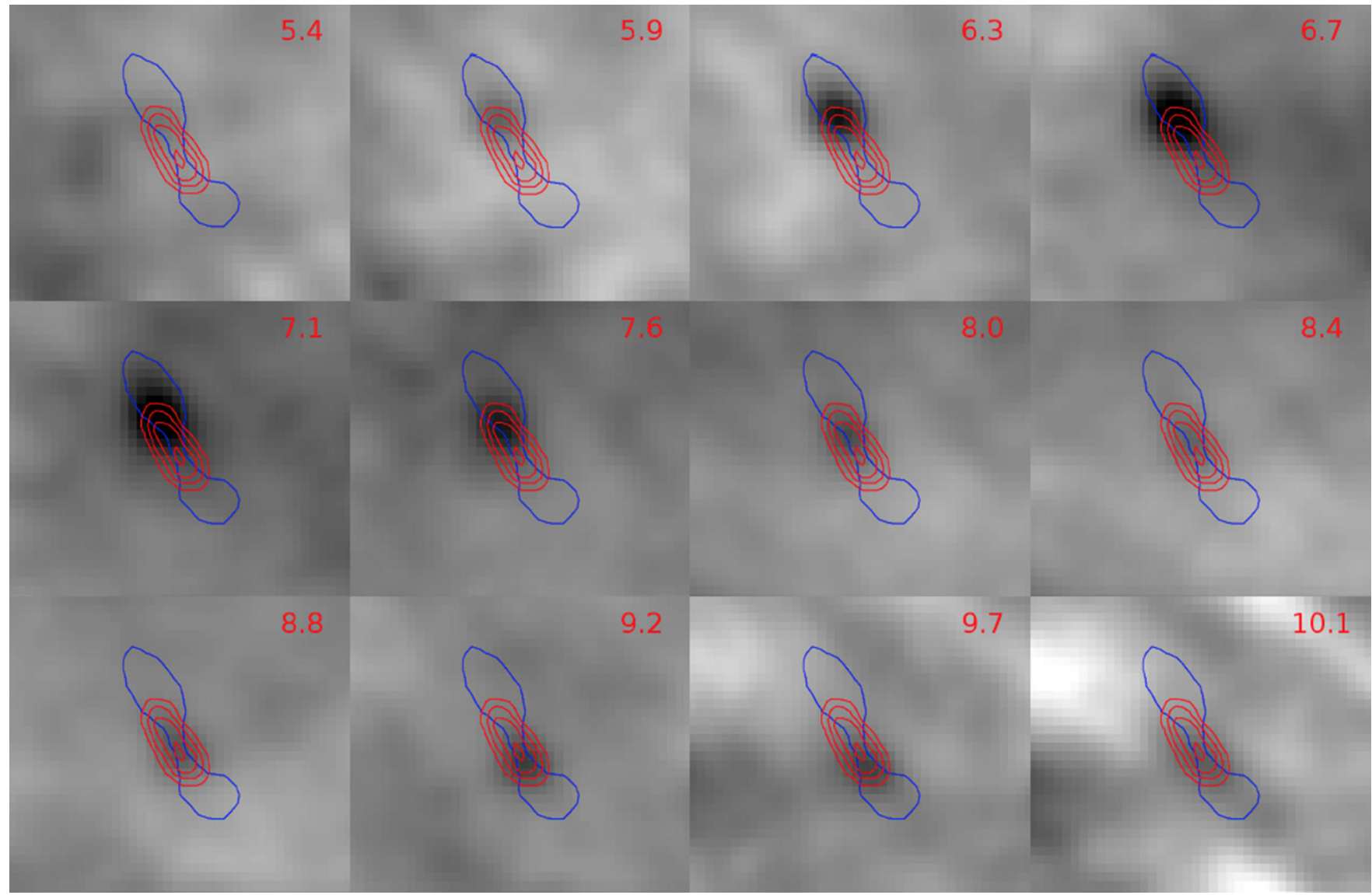

Figure 4. Grid of images showing the 114-426 disk in the CO $J=3-2$ line (gray scale). The data values shown range over -1.0 to $+1.0 \mathrm{Jy}^{-1}$ beam ${ }^{-1}$. The LSR radial velocity of the center of each channel is indicated in the upper right in $\mathrm{km} \mathrm{s}^{-1}$. The blue contour shows the outline of the disk in the HST F775W filter. The red contours show the $856 \mu \mathrm{m}$ dust continuum emission with the same levels as in the top panel in Figure 2.

Table 2

${ }^{12} \mathrm{CO}$ Absorption Radial Velocities and Locations in the d114-426 Disk

\begin{tabular}{|c|c|c|c|c|c|c|}
\hline $\begin{array}{l}V_{\mathrm{LSR}} \\
\left(\mathrm{km} \mathrm{s}^{-1}\right)\end{array}$ & $\begin{array}{c}\alpha \\
\mathrm{J} 2000\end{array}$ & $\begin{array}{c}\delta \\
\mathrm{J} 2000\end{array}$ & $\begin{array}{c}I(\mathrm{CO})^{\mathrm{a}} \\
(\mathrm{Jy})\end{array}$ & $\begin{array}{l}\Delta T(\mathrm{CO})^{\mathrm{a}} \\
\quad(\mathrm{K})\end{array}$ & $\begin{array}{c}T_{\text {bck }}{ }^{b} \\
(\mathrm{~K})\end{array}$ & $\frac{\overline{\Delta T(\mathrm{CO})}}{T_{\mathrm{bck}}}$ \\
\hline 5.43 & 053511.332 & -052426.10 & -0.07 & -3.0 & 51 & 0.06 \\
\hline 5.86 & 053511.328 & -052426.04 & -0.14 & -6.0 & 59 & 0.10 \\
\hline 6.28 & 053511.336 & -052426.04 & -0.43 & -18.4 & 67 & 0.27 \\
\hline 6.70 & 053511.336 & -052426.04 & -0.61 & -26.1 & 73 & 0.36 \\
\hline 7.13 & 053511.334 & -052426.09 & -0.63 & -27.0 & 78 & 0.35 \\
\hline 7.55 & 053511.331 & -052426.20 & -0.42 & -18.0 & 82 & 0.22 \\
\hline 7.97 & 053511.326 & -052426.31 & -0.24 & -10.3 & 82 & 0.13 \\
\hline 8.40 & 053511.322 & -052426.38 & -0.14 & -6.0 & 81 & 0.07 \\
\hline 8.82 & 053511.314 & -052426.59 & -0.18 & -7.7 & 77 & 0.10 \\
\hline 9.24 & 053511.310 & -052426.72 & -0.28 & -12.0 & 71 & 0.17 \\
\hline 9.67 & 053511.306 & -052426.81 & -0.26 & -11.1 & 63 & 0.17 \\
\hline 10.09 & 053511.306 & -052426.81 & -0.15 & -6.4 & 51 & 0.13 \\
\hline 10.51 & 053511.306 & -052426.81 & -0.05 & -2.1 & 39 & 0.05 \\
\hline
\end{tabular}

Note.

${ }^{a} I(\mathrm{CO})$ is the ALMA-DETECTED $J=3-2$ absorption in Janskys; $T(\mathrm{CO})$ is the absorption in brightness temperature units.

$\mathrm{b}$ The background is estimated by extrapolation of the ${ }^{12} \mathrm{CO}$ emission in the $J=1-0$ emission from Shimajiri et al. (2011) and the $J=2-1$ emission from Berne et al. (2014).

frequency channel is zero. Small-scale $\left(<5^{\prime \prime}\right)$ emission produces compact positive signals surrounded by extended (larger than $\left.5^{\prime \prime}\right)$ negative bowls. Compact $\left(<5^{\prime \prime}\right)$ absorption features with amplitudes larger than the small-scale intensity variations in the background are retained in the maps as compact regions with negative values. In the velocity range occupied by the strong background emission, the $\mathrm{CO}$ intensity fluctuations in the ALMA data cube have a $1 \sigma \mathrm{rms}$ of about 0.1 Jy or less $(\sim 4 \mathrm{~K})$ in apertures ranging in diameter from 0 ". 5 to $5^{\prime \prime}$, less than $10 \%$ of the single-dish surface brightness in the 
background line core against which the $\mathrm{CO}$ absorption from 114-426 is seen, indicating that the background emission from the Orion A cloud is relatively smooth on these angular scales and does not contain intensity variations as large as the absorption signal from 114-426.

The northern portion of the 114-426 disk (as traced by dustcontinuum) has the strongest $\mathrm{CO}$ absorption with a depth of $-0.63 \mathrm{Jy} \mathrm{beam}^{-1}$. The southern lobe of the dust continuum disk has a CO absorption depth of $-0.28 \mathrm{Jy}^{\text {beam }}{ }^{-1}$ (Figure 3 ). These flux deficits correspond to temperatures of 27 and $12 \mathrm{~K}$ below the background $\mathrm{CO}$ emission. Table 2 lists the location of the deepest absorption as a function of radial velocity, the absorption depth in Janskys and degrees Kelvin, the extrapolated single dish data at the location of 114-426 interpolated to the radial-velocities of the ALMA data, and the ratio of the absorption divided by the estimated background $\mathrm{CO}$ temperature. Figure 4 shows the CO data cube (gray-scale) with the outline of the visual silhouette shown as a blue contour and the dust continuum emission shown in red contours. The spatial extent of the deepest, blueshifted $\mathrm{CO}$ absorption from the northeast part of the disk appears to be wider than the $\sim 0$ " 5 synthesized ALMA beam and wider than the dust layer seen in the HST images, possibly indicating the presence of $\mathrm{CO}$ above and below the dust responsible for the visual-wavelength silhouette. The deepest absorption in the northern portion of the disk occurs at $6.7 \pm 0.15 \mathrm{~km} \mathrm{~s}^{-1}$. The deepest absorption in the southern portion of the disk occurs at $9.6 \pm 0.15 \mathrm{~km} \mathrm{~s}^{-1}$. The two deepest absorption peaks differ in radial velocity by $\Delta V=2.9 \pm 0.2 \mathrm{~km} \mathrm{~s}^{-1}$ and their centroids differ in position by $\Delta X=0 " .86 \pm 0^{\prime \prime} .1$.

\section{DISCUSSION}

The observed $\mathrm{CO}$ absorption can be affected by several factors including the optical depth, radial velocity, and temperature of the disk gas, beam dilution since the disk silhouette is thinner along its minor axis than the ALMA synthesized beam, and the brightness temperature distribution of the background emission as a function of radial velocity.

The projected 114-426 disk thickness varies from less than $z_{d}=0$ ". 09-0". 43 in the F775W image (Figure 2). The smaller value, however, corresponds to only the foreground portion of the disk which occults the central reflection nebula. CO absorption against the background cloud samples the entire line of sight (LOS) through the disk, including the portion behind the reflection nebula. The projected width of the deepest $\mathrm{CO}$ absorption at $7.1 \mathrm{~km} \mathrm{~s}^{-1}$ (cyan contour in Figure 2 and the panel labeled 7.1 in Figure 4) may be marginally wider than the 0 ". 43 apparent thickness of the silhouette. As stated above, it is possible that optically thick $\mathrm{CO}$ extends above and below the region seen in silhouette in the $H S T$ images.

Emission and absorption from the disk in the $r_{\mathrm{B}} \sim$ 0 . 25 radius synthesized ALMA beam is diluted by a factor $f_{\mathrm{B}} \approx 2 r_{\mathrm{B}} z_{\mathrm{CO}} / \pi r_{\mathrm{B}}^{2}=2 z_{\mathrm{CO}} / \pi r_{\mathrm{B}} \sim 0.5-1$. At the location of the strongest $\mathrm{CO}$ absorption in the northeast, the thickness in the F775W HST image is $z_{d} \approx 0^{\prime \prime} .43$ and $f_{\mathrm{B}} \approx 1$. At the weaker southwest absorption, $z_{d} \approx 0$ ". 20 and $f_{\mathrm{B}} \approx 0.5$. Correcting the southwest dip for beam dilution makes its absorption depth comparable to the northeast depth. The inner part of the disk where the highest rotation speeds are expected may be more beam diluted because the disk is expected to be even thinner. Additionally, the highest velocity CO in the center of the disk may extend beyond the velocity range where the background emission is brighter than the disk emission, in which case it would not be seen in absorption and any emission signature may be too beam-diluted to be seen.

The mean volume and column density of the 114-426 disk can be estimated from the dust-continuum derived mass, $M_{\text {disk }}=5.9 \times 10^{30} \mathrm{~g}$, and the projected size of the submillimeter dust emission, $\sim 0^{\prime \prime}$. 2 by $1^{\prime \prime}$. Assuming that the disk mass is distributed uniformly in a pill-shaped volume with these dimensions implies a mean density, $n\left(\mathrm{H}_{2}\right)>10^{7} \mathrm{~cm}^{-3}$ and column density, $N\left(\mathrm{H}_{2}\right)>4 \times 10^{22} \mathrm{~cm}^{-2}$. The low- $J$ transitions of $\mathrm{CO}$ will be thermalized at the gas temperature, and unless it is depleted by more than three orders of magnitude compared to molecular clouds, it will be optically thick.

The apparent surface brightness of the $856 \mu \mathrm{m}$ continuum in the ALMA beam, $\approx 4 \mathrm{mJy}$ beam $^{-1}$, is also affected by beam dilution. Using the beam dilution factor appropriate to the inner $350 \mathrm{AU}$ portion of the disk, $f_{\mathrm{B}} \sim 0.5-1$, implies an average surface brightness of $S_{\nu} \sim 8-4 \mathrm{mJy}$ if the disk were to fill the beam. Comparing this brightness to the Planck function, $B_{\nu}(T)$ for temperatures ranging from $T_{\text {dust }}=20-40 \mathrm{~K}$ from a solidangle equal to the synthesized ALMA beam provides an estimate of the dust optical depth, $\tau=S_{\nu} / B_{\nu}(T)$. The mean optical depth of the dust ranges from $\tau=0.016$ to 0.032 for $20 \mathrm{~K}$ dust or $\tau=0.006$ to 0.013 for $40 \mathrm{~K}$ dust.

A surprising feature of the 114-426 dust continuum emission is the sharp drop in flux at a radius of about $200 \mathrm{AU}$ from the suspected location of the central star (Figures 1 and 2). The flux decreases by $3 \mathrm{mJy} \mathrm{beam}^{-1}$ in a region comparable to or smaller than the synthesized beam diameter. The outer parts of the disk beyond $R \sim 200$ AU but interior to the edge at visual wavelengths $(1$ ". 4 or $580 \mathrm{AU}$ northeast of the suspected location of the central star and 0 ". 8 or 330 AU to the southwest) do not exhibit any continuum emission above the noise. Assuming that the total area of the outer region of the disk is 0.4 square arcseconds based on a comparison between the area of the silhouette in the HST images and the area where $856 \mu \mathrm{m}$ emission is detected, the $0.6 \mathrm{mJy}$ beam $^{-1}$ sensitivity limit implies that the column density of $\mathrm{H}_{2}$ must be $N\left(\mathrm{H}_{2}\right)<8 \times 10^{21} \mathrm{~cm}^{-3}$. This column corresponds to a visual-wavelength extinction of about eight magnitudes, assuming normal ISM dust. The HST images show that the visual extinction through the outer disk ranges from about $0.5 \mathrm{mag}$ in the translucent northeastern edge to more than three magnitudes at $R \sim 200 \mathrm{AU}$. Thus, for normal ISM dust, the visual extinction implies that the submillimeter continuum ought to be detected at a level of $\sim 0.2 \mathrm{mJy} \mathrm{beam}^{-1}$ for a $0^{\prime \prime} .5$ beam diameter.

At the two velocities where the deepest $\mathrm{CO}$ absorption is seen $\left(6.9\right.$ and $\left.9.5 \mathrm{~km} \mathrm{~s}^{-1}\right)$, the background $\mathrm{CO}$ line temperature is between 60 and $70 \mathrm{~K}$. Assuming that the absorbing layer is optically thick and beam-filling (as it may be at the northwest portion of 114-426), an absorption depth of $-0.6 \mathrm{Jy} \mathrm{beam}^{-1}$ against a $60-70 \mathrm{~K}$ background corresponds to a brightness temperature of $33-43 \mathrm{~K}$. If the $\mathrm{CO}$ absorption is beam-diluted by a factor $f_{\mathrm{B}}$, the temperature will be correspondingly lower and likely a good estimate of the gas temperature where the $\mathrm{CO}$ optical depth reaches a value of order unity.

The CO absorption has a similar spatial extent as the dust continuum along the disk major axis. If the absorption minima trace the peak orbit speeds at their centroid locations, the mass of the central star can be estimated since the 114-426 disk is within a few degrees of being edge-on. As discussed above, the deepest 
absorptions occur at a projected separation of $\Delta X=$ $0.86 \pm 0.1, \quad$ implying $\quad R \approx 178 \pm 20 \mathrm{AU}$ $\left(2.7 \pm 0.3 \times 10^{15} \mathrm{~cm}\right)$ at a distance of $414 \mathrm{pc}$. The radial velocity difference between the north and south absorption dips, $\Delta V=2.9 \pm 0.2 \mathrm{~km} \mathrm{~s}^{-1}$, implies $V_{\text {orbit }}(R)=1.45 \pm 0.1$ $\mathrm{km} \mathrm{s}^{-1}$. Thus, assuming that the disk is in Keplerian rotaion, the enclosed mass is $M_{*}=R V_{\text {orbit }}^{2}(R) / G \sim 0.43 \pm 0.11 M_{\odot}$. This estimate may be a lower bound since $\mathrm{CO}$ absorption would not trace gas outside the velocity range $V_{\mathrm{LSR}}=5-10 \mathrm{~km} \mathrm{~s}^{-1}$ where the brightness temperature of the background $\mathrm{CO}$ emission drops below the brightness temperature of the $\mathrm{CO}$ in the disk. The absence of emission at larger radial velocities, however, also sets an upper bound on the stellar mass.

We modeled the $\mathrm{CO}$ absorption from a geometrically thin, optically thick Keplerian disk inclined by a few degrees, convolved with a Gaussian to represent the synthesized ALMA beam using two free parameters: an inner hole with radius $R_{\text {in }}$ and the central star mass, $M_{*}$. If the inner hole is small, a stellar mass between $0.4 \mathrm{msol}$ and $0.7 M_{\odot}$ is favored. A stellar mass of about $1 M_{\odot}$ and a thin annular disk with a large $(\sim 250 \mathrm{AU})$ hole, however, is also allowed. A flaring disk with a small scale-height close the star would produce results similar to a model disk with a large inner hole because of radially dependent beam-dilution.

Our mass estimate is lower than than $1.5 M_{\odot}$ estimated by McCaughrean et al. (1998) based on extrapolation of the observed brightness of the reflection nebula. More efficient scattering by large, high-albedo grains, and significant forward scattering would lower the estimated intrinsic magnitude of the central star, and the resulting stellar mass estimate.

$\mathrm{HCO}^{+}, \mathrm{HCN}$, and $\mathrm{CS}$ lines, in either emission or absorption, are not detected from 114-426. Their brightness temperatures may be either similar to that of the background, or these species may be depleted. Given the faint dust continuum emission, and the low relative abundances of these species compared to $\mathrm{CO}$ in molecular clouds (Bergin \& Tafalla 2007), their absence in 114-426 may not be that surprising.

\subsection{Is 114-426 an Evolved Proto-planetary Disk?}

The small estimated mass of the 114-426 disk is in stark contrast to its large size in visual wavelength images. Visual extinction is dominated by small grains while the submillimeter emission is more sensitive to large particles. Multi-wavelength observations of circumstellar disks indicate that the grain size decreases with increasing distance from the central star. These observations suggest that the outermost regions of young circumstellar disks might be populated by dust similar to the ISM (Testi et al. 2014).

Photometry of the translucent northeast outer edge of the 114-426 disk has shown that the extinction is gray, implying that the mean particle size responsible for the attenuation of background light is large compared to interstellar grains in the outer parts of the disk at a projected radius of 400-500 AU from its central star (Throop et al. 2001). Reddening between 2 and $4 \mu \mathrm{m}$ (Shuping et al. 2003), however, indicates that the grains must be smaller than a few micro-meters. These observations suggest that the 114-426 is an evolved disk in which solids and ices have been incorporated into large particles. These observations, and comparison to data on young disks (Testi et al. 2014), suggests that 114-426 is an evolved disk.
Grain growth rates are expected to increase with decreasing distance from the central star. Thus, it is possible that in the inner portions of the 114-426 disk, particles have grown to sizes larger than a millimeter, in which case the submillimeter emissivity per unit mass of solids (and gas) would decrease. Thus, the assumptions used above for converting the observed continuum flux into dust and gas mass may not apply. In this case, our estimated disk mass would be a lower bound. If the mean particle size responsible for the continuum emission is larger than the wavelength, however, then the shape of the dust continuum spectrum should approach the Rayleigh-Jeans slope of -2 . Future subarcsecond angular resolution, multi-frequency observations with ALMA can directly measure the submillimeter continuum spectral index to determine if indeed grains are larger than the observing wavelength. Given that the 114-426 disk lacks evidence for being surrounded by a photoionized skin, spectral index measurements can be extended into the centimeter-wavelength domain using the JVLA. Such measurements may in the future provide direct evidence for the sequestration of solids and ices into large bodies, a process expected to be the first phase of planet formation.

It is possible that the absence of dense gas tracers in the 114-426 disk is also evidence for chemical evolution. In the low-temperature conditions encountered in the outer Orion Nebula, many common tracers of molecular gas may have frozen out of the gas phase and locked into icy grain mantles. UV and X-ray photolysis of light organic compounds in an ice matrix may lead to their conversion into heavy organic material. Thus, the absence of measurable HCN, CS, and $\mathrm{HCO}^{+}$lines may also be an indication that the 114-426 disk is highly evolved. Sensitive searches for other compounds in the submillimeter spectrum of 114-426 with ALMA should test this hypothesis.

Miotello et al. (2012) proposed that 114-426 is being photoablated by the non-ionizing FUV radiation field in the Orion Nebula and suggested a mass-loss rate $\dot{M}_{\text {disk }} \sim(3-5) \times 10^{-7}$ $M_{\odot} \mathrm{yr}^{-1}$. For the disk mass estimated above, $5.9 \times 10^{30} \mathrm{~g}$, the disk lifetime is $\tau \sim M_{\text {disk }} / \dot{M}_{\text {disk }} \sim(1.0-1.6) \times 10^{4}$ years.

\subsection{Comparison to ALMA Observations of Other Silhouette Disks in Orion}

Two other giant disks in Orion, 216-0939 near OMC2, and 253-1536 in M43 show strong dust continuum (Mann et al. 2014) and bright line-emission in the dense gas tracers. CO is seen both in emission and absorption, but the latter signal is less prominent than in 114-426, probably because the background $\mathrm{CO}$ emission is dimmer, and possibly because foreground $\mathrm{CO}$ is more likely to be present along the LOS. Analysis of the displacements and Doppler shifts of the absorption signals give stellar mass estimates comparable to those derived from the emission line rotation curves, namely $3.5 M_{\odot}$ for 253-1536 (Williams et al. 2014) and $1.1 M_{\odot}$ for 216-0939 (in preparation). These objects have central star masses around $1 M_{\odot}-3 M_{\odot}$ and are surrounded by massive disks exhibiting strong submillimeter dust continuum and bright line emission in $\mathrm{HCN}, \mathrm{CS}$, and $\mathrm{HCO}^{+}$. The dim dust continuum and lack of detection of the dense gas tracers in the 114-426 silhouette disk is consistent with the low mass estimated for its central star and surrounding disk (Andrews et al. 2013). 


\subsection{Other Objects in the 114-426 ALMA Field}

Figure 1 shows that the northern portion of the 114-426 ALMA field contains bright $\mathrm{CO}$ emission associated with the $\mathrm{HH}$ object HH 530 (Bally et al. 2000). The $\mathrm{H} \alpha$ image shows a jet-like feature east of the star V2202 Ori (source 4 in Table 1 and the figures; source 106-417 in O'Dell \& Wen 1994). Many components of $\mathrm{HH}$ 530, however, exhibit supersonic motions toward the southwest with velocities ranging from 25 to $70 \mathrm{~km} \mathrm{~s}^{-1}$ (O'Dell $\&$ Doi 2003), indicating that these shocks trace a background outflow from a source embedded in the vicinity of OMC1-south, northeast of the 114-426 field. Thus, V2202 Ori is not related to this flow. The $\mathrm{CO}$ emission in the vicinity of $\mathrm{HH} 530$ extends from $V_{\mathrm{LSR}}=+2$ to over $+20 \mathrm{~km} \mathrm{~s}^{-1}$ and may be associated with $\mathrm{HH}$ 530. It may trace a shell swept-up by this externally irradiated $\mathrm{HH}$ object. Given that both red and blueshifted components of the flow are seen in the same lobe (with respect to the $V_{\mathrm{LSR}} \sim 9 \mathrm{~km} \mathrm{~s}^{-1}$ radial velocity of the background $\mathrm{CO}$ emission in this portion of Orion), the outflow must move close to the plane of the sky. The presence of molecules in $\mathrm{HH} 530$ shows that the $\mathrm{H} \alpha$ emission seen in the HST images must trace the ionized skin of a molecular outflow, indicating that this flow is only partially photo-ionized by the UV radiation field in the Orion Nebula.

The prominent bow-shaped $\mathrm{H} \alpha$ structure located about $6^{\prime \prime}-$ $10^{\prime \prime}$ west of 114-426 is not associated with any CO features. This bow, however, also exhibits a large proper motion of about $40 \mathrm{~km} \mathrm{~s}^{-1}$ toward the southwest as shown by the multi-epoch HST images of O'Dell \& Doi (2003). It is unclear if this feature is associated with an outflow from 114-426 or an other unrelated background flow similar to HH 530 but without a CO counterpart.

\section{CONCLUSIONS}

The largest disk seen in silhouette against the visualwavelength emission of the Orion Nebula, 114-426, is detected in dust continuum emission, and in absorption in the $345 \mathrm{GHz}$ $J=3-2 \mathrm{CO}$ transition. The $\mathrm{CO}$ absorption against the warm background emission from the Orion A cloud has the same spatial extent and morphology as the dust continuum emission. The disk in these tracers is a factor of two smaller than in the visual wavelength $H S T$ images, however. A model consisting of a Keplerian rotation curve in a geometrically thin, edge-on disk convolved with a 0 ". 5 Gaussian beam-profile indicates that $M_{*}$ is less than $1 M_{\odot}$ with a most likely value around $0.4 M_{\odot}$. The disk is not detected in $\mathrm{HCN}, \mathrm{CS}$, or $\mathrm{HCO}^{+}$lines.

Assuming a gas-to-dust ratio of 100, and a dust temperature of $20 \mathrm{~K}$, the observed submillimeter dust continuum implies a disk mass of $3.1 \pm 0.6$ times the mass of Jupiter $\left(5.9 \pm 1.1 \times 10^{30} \mathrm{~g}\right)$. Comparison with ALMA observations of two other large disks in the Orion region provides support for a low-stellar mass and low-disk mass.
This research of J.B. was in part supported by National Science Foundation (NSF) grant AST-1009847. D.J. acknowledges support from a Natural Sciences and Engineering Research Council (NSERC) Discovery Grant. This paper uses ALMA data obtained with program ADS/JAO.ALMA\#2011.0.00028.S. ALMA is a partnership of the European Southern Observatory (ESO) representing member states, Associated Universities Incorporated (AUI) and the National Radio Astronomy Observatories (NRAO) for the National Science Foundation (NSF) in the USA, NINS in Japan, NRC in Canada, and NSC and ASIAA in Taiwan, in cooperation with the Republic of Chile. The Joint ALMA Observatory (JAO) is operated by ESO (Europe), AUI/ NRAO (USA), and NAOJ (Japan). The National Radio Astronomy Observatory is a facility of the National Science Foundation operated under cooperative agreement by Associated Universities, Inc.

\section{REFERENCES}

Andrews, S. M., Rosenfeld, K. A., Kraus, A. L., \& Wilner, D. J. 2013, ApJ, 771,129

Bally, J., Licht, D., Smith, N., \& Walawender, J. 2006, AJ, 131, 473

Bally, J., O’Dell, C. R., \& McCaughrean, M. J. 2000, AJ, 119, 2919

Bally, J., Testi, L., Sargent, A., \& Carlstrom, J. 1998, AJ, 116, 854

Bergin, E. A., \& Tafalla, M. 2007, ARA\&A, 45, 339

Berne, O., Marcelino, N., \& Cernicharo, J. 2014, arXiv:1408.2999

Eisner, J. A., \& Carpenter, J. M. 2006, ApJ, 641, 1162

Eisner, J. A., Plambeck, R. L., Carpenter, J. M., et al. 2008, ApJ, 683, 304

Johnstone, D., Hollenbach, D., \& Bally, J. 1998, ApJ, 499, 758

Mann, R. K., Di Francesco, J., Johnstone, D., et al. 2014, ApJ, 784, 82

Mann, R. K., \& Williams, J. P. 2009, ApJL, 694, L36

Mann, R. K., \& Williams, J. P. 2010, ApJ, 725, 430

McCaughrean, M. J., Chen, H., Bally, J., et al. 1998, ApJL, 492, L157

McCaughrean, M. J., \& O’Dell, C. R. 1996, AJ, 111, 1977

Menten, K. M., Reid, M. J., Forbrich, J., \& Brunthaler, A. 2007, A\&A, 474, 515

Miotello, A., Robberto, M., Potenza, M. A. C., \& Ricci, L. 2012, ApJ, 757, 78 O'Dell, C. R. 2001, ARA\&A, 39, 99

O’Dell, C. R., \& Doi, T. 2003, AJ, 125, 277

O’Dell, C. R., \& Wen, Z. 1994, ApJ, 436, 194

Peng, T.-C., Wyrowski, F., Zapata, L. A., Güsten, R., \& Menten, K. M. 2012, A\&A, 538, A12

Ricci, L., Robberto, M., \& Soderblom, D. R. 2008, AJ, 136, 2136

Robberto, M., Soderblom, D. R., Bergeron, E., et al. 2013, ApJS, 207, 10

Shimajiri, Y., Kawabe, R., Takakuwa, S., et al. 2011, PASJ, 63, 105

Shimajiri, Y., Kitamura, Y., Saito, M., et al. 2014, A\&A, 564, A68

Shuping, R. Y., Bally, J., Morris, M., \& Throop, H. 2003, ApJL, 587, L109

Testi, L., Birnstiel, T., Ricci, L., et al. 2014, in Protostars and Planets VI, ed. H. Beuther, R. S. Klessen, C. P. Dullemond, \& T. Henning (Tucson, AZ: Univ. Arizona Press), 339

Throop, H. B., Bally, J., Esposito, L. W., \& McCaughrean, M. J. 2001, Sci, 292, 1686

Williams, J. P., Andrews, S. M., \& Wilner, D. J. 2005, ApJ, 634, 495

Williams, J. P., \& Best, W. M. J. 2014, ApJ, 788, 59

Williams, J. P., Mann, R. K., Di Francesco, J., et al. 2014, ApJ, 796, 120 